\title{
Ophthalmology in Luanda (Angola): a hospital based report
}

\author{
F J Carreras, F Rodríguez-Hurtado, H David
}

\begin{abstract}
Aims/Background-As part of a 4 year Spanish development aid programme, an ophthalmic hospital was set up in Luanda in 1991 for the in situ training of local ophthalmologists. Presented here are the data obtained from 4201 patients treated during the first 2 years of the project.

Methods-Patients were referred to the institute from the emergency ward at the Luanda General Hospital, selected mainly according to the severity of their disease. The following data were collected from the clinical reports: age, sex, diagnosis (single or multiple), type of treatment (medical or surgical), acuity of the best eye at the time of diagnosis, and main disease group.

Results-The main causes of blindness treated were: cataracts; glaucoma; optic nerve diseases (neuritis and atrophy); trauma; xerophthalmia; uveitis; hereditary retinal diseases (degenerative myopia, retinitis pigmentosa, albinism, and Stargardt's disease); retinal detachment; and diabetic retinopathy.

Conclusions-Sanitary resources in Angola are generally inadequate, and ophthalmic care is no exception to this. Owing to the high percentage of preventable or treatable blinding diseases in this environment, a campaign of social education should always be held along with any medical programme, in order to optimise the available resources.

(Br f Ophthalmol 1995; 79: 926-933)
\end{abstract}

The population of Angola is officially estimated at six million, although the true figure may now be substantially less, as the country has seen 30 years of active fighting, which started with the struggles for independence and has continued to date as open civil war. Luanda, the capital, was designed by the Portuguese colonists for a population of half a million; today, as a result of the influx of refugees, there are over two million inhabitants.

The typical distribution pattern of medical facilities and human resources found in most African countries, with most hospitals and doctors being concentrated in large cities, is especially marked in Angola. When the project described in this paper began, there were only three Angolan ophthalmologists in Luanda, working alongside half a dozen aid workers from communist or former communist countries such as Cuba, Vietnam, and Russia. Outside the capital there were no ophthalmic specialists at all.
As part of a Spanish development aid programme, an ophthalmic hospital was set up in Luanda. The aim was for two Spanish ophthalmologists and one Angolan specialist to train new ophthalmologists and optometry specialists. The hospital was equipped with 20 beds and two operating theatres, with auxiliary electrical and water supplies available in case of emergency.

During the first phase of the project all types of basic ophthalmic surgery were performed, except for intravitreal surgery and corneal grafts. Cataract surgery included both extra and intracapsular procedures, but intraocular lens implants were only performed in the third year of the project. Also in the third year, argon and YAG laser therapies were added to the range of services. On site pharmacy facilities ensured the availability of the treatments prescribed.

Owing to the persistent fighting and shortage of human resources it would not have been practical to undertake a study of the prevalence of ophthalmic diseases in Angola. The aim of the project was not to provide direct care for the population at large, but rather to train ophthalmologists who could later spread throughout the country in order to address the healthcare needs of the people.

Ophthalmological diagnosis requires considerable expertise and the use of sophisticated equipment. Both requirements were fulfilled at the Ophthalmic Institute in Luanda, for the first time in 30 years.

In this paper the data obtained from more than 4000 patients treated during the first 2 years of the project (starting in October 1991) are presented. Although the data cannot be extrapolated to the general population, the information they provide should be of value for future planners of hospital resources in similar projects in Angola and neighbouring countries.

\section{Materials and methods}

\section{SELECTION OF PATIENTS}

Patients were selected twice weekly from the emergency ward at the Luanda General Hospital. The institute acted only as a centre for referrals rather than as an emergency ward. This decision was taken in view of the limited availability of staff able to provide ophthalmic assistance at the beginning of the project. The institute thus maintained its teaching character and avoided the risk of being overwhelmed by the needs of the general population. An average of 70 patients per day were treated twice a week in the emergency ward (that is, $100 \%$ capacity). A team of one ophthalmologist and 
four residents carried out preliminary diagnosis and treatment and selected cases for referral to the Ophthalmic Institute. The team operated on surgical emergencies the same afternoon and the following day. Owing to the limited capacity of the institute, a number of banal pathologies - mainly conjunctivitis and blepharitis - were treated in the general hospital and not recorded in the institute's files. The criteria used for selecting patients to be referred to the institute were mainly the severity of the disease or its relevance to the teaching programme. The institute also received patients from the optometry section of the general hospital. Most patients were civilians, since the institute did not usually treat military casualties.

During the first 2 years of the project, 4201 patients were treated at the institute and included in the present study.

\section{CLINICAL METHODS}

Patients were treated by appointment at the Ophthalmic Institute by the team that had first examined them in the emergency ward at the general hospital. An individual case file was opened and the patient's clinical history and general examination findings were recorded. The standard examination consisted of: inspection of the general complexion (face, brows, eyelids); corneal reflex position; ocular movements and cover test; pupil size and light reflex; manual keratometry and streak retinoscopy; determination of the best visual acuity; slit-lamp examination of the anterior segment; Goldmann applanation tonometry; and direct, and frequently indirect, ophthalmoscopy. Optional explorations, such as Goldmann perimetry or Hertel exophthalmometry, were carried out only when required by the previous findings.

\section{DATA COLLECTION}

The following data were collected from the clinical reports: age; sex; diagnoses (single or multiple); type of treatment (medical or surgical); acuity of the best eye at the time of diagnosis; main disease group (cornea, retina, glaucoma, etc).

All diagnoses were classified with a three letter code and grouped into one of the following categories: (1) lids; (2) orbit; (3) lacrimal system; (4) conjunctiva; (5) cornea-sclera; (6) uveitis-endophthalmitis; (7) glaucoma; (8) lens; (9) retinal and choroidal diseases other than uveitis; (10) squint; (11) neuroophthalmology; (12) syndromes-congenital anomalies; (13) ametropies; (14) trauma.

Duplication of diagnoses was avoided such that myopia magna, for instance, appears only among retinal diseases and not among ametropies. Nevertheless, multiple categorisation of the same patient did occur on occasion, if the pathological process presented by the eye was not covered with a single diagnosis. For example, secondary cataracts were primarily included among cataracts, and secondarily among trauma or uveitis, if the cause of the cataractogenic process could be inferred with a sufficient degree of certainty. Unfortunately this was not always the case, as many patients in this environment commonly attribute all their diseases to old traumas. A typical case would be that of iridocyclitis with high intraocular pressure, which we recorded under both secondary glaucoma and uveitis. If such a case were to be found once the active disease had passed however - with a tranquil eye and unilateral optic atrophy with excavation, with or without elevated intraocular pressure - no attempt would be made to attribute the unilateral neuropathy to an unobserved uveitis.

As the number of different diagnoses was high, each category was divided into single diagnoses or groups of closely related diseases, so as to present less than 15 groups per category. Data management was carried out with an Apple Macintosh Quadra 650 computer using SYSTAT 5.0 software (from Systat Co).

\section{DEFINITIONS}

Following the definition of the World Health Organisation, ${ }^{12}$ we considered as blind those patients with a corrected visual acuity in the better eye of less than $3 / 60(10 / 200)$, and as visually impaired (significant visual loss) those with visual acuity in the better eye of less than $6 / 18$. Only bilateral blindness and visual loss were considered in this study.

\section{Results}

Results are summarised in Table 1 and Figures 1-10. Figure 1 shows the distribution of patients across the 14 categories considered. Each category has been broken down to show the main diseases in each group. Distribution by age and sex is also shown for the main categories.

We studied 4201 patients, 2363 of whom had an ophthalmic disease, the remainder being only ametropies. Of these, $49 \cdot 2 \%$ were female and $50 \cdot 8 \%$ male.

BLINDNESS AND SIGNIFICANT VISUAL LOSS A total of 265 of our patients were blind according to the definition of the World Health Organisation. ${ }^{12}$ Patients were considered as blind if their corrected visual acuity in the better eye was less than $3 / 60$. In this group, 111 were female $(41.9 \%)$ and 154 male

Table 1 Main causes of blindness at the Ophthalmic Institute of Luanda

\begin{tabular}{lcr}
\hline Main diagnosis & Frequency & $\%$ \\
\hline Cataract & 64 & $24 \cdot 2$ \\
Glaucoma & 55 & $20 \cdot 9$ \\
Optic atrophy & 24 & $9 \cdot 1$ \\
Hereditary retinal disease & 22 & $8 \cdot 3$ \\
Trauma & 20 & $7 \cdot 6$ \\
Optic neuritis & 11 & $4 \cdot 1$ \\
Xerophthalmia & 11 & $4 \cdot 1$ \\
Uveitis & 10 & $3 \cdot 8$ \\
Retinal detachment & 8 & $3 \cdot 0$ \\
Diabetic retinopathy & 3 & $1 \cdot 1$ \\
Other & 37 & $13 \cdot 9$ \\
Total & 265 & 100 \\
\hline
\end{tabular}



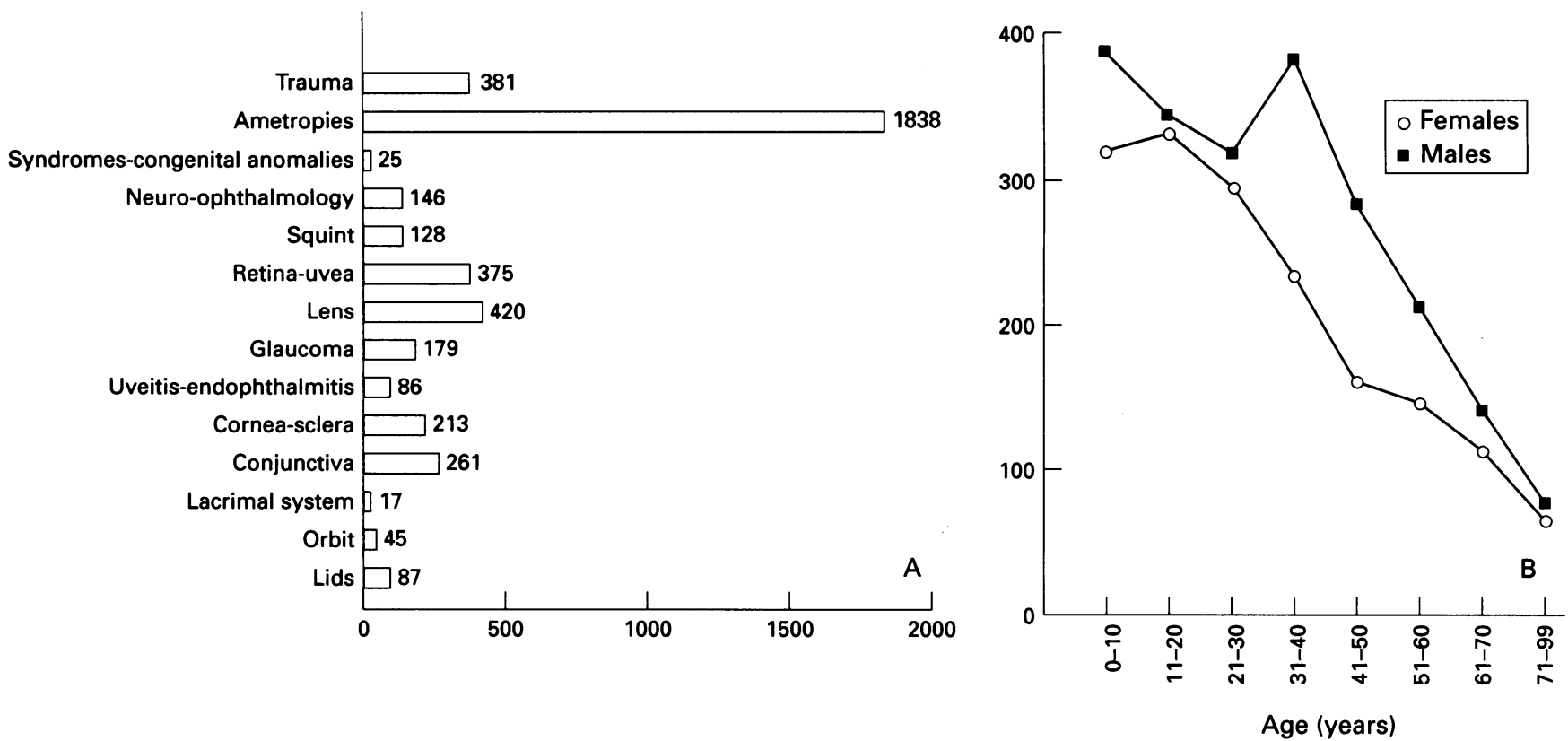

Figure 1 (A) Distribution of 4201 patients across the 14 categories considered. (B) Distribution of all pathologies by age and sex.

$(58 \cdot 1 \%) ; 127(47 \cdot 1 \%)$ were totally blind - that is, with no light perception whatsoever. When ametropies were discounted and only 2363 patients with pathologies considered, the blind accounted for $11 \cdot 2 \%$ of all pathologies.

Patients were considered as visually impaired if their visual acuity was less than $6 / 18$ in the better eye. ${ }^{12}$ In our series a total of 159 patients presented with significant visual loss, 60 of whom were female $(37 \cdot 7 \%)$ and 99 male $(62 \cdot 3 \%)$. Both groups totalled 424 with blindness or severely reduced visual acuity that is, $17 \cdot 9 \%$ of all the pathologies.

In the blind group, 80 patients (30.2\%) received surgical treatment. The remaining $185(69 \cdot 8 \%)$ were limited to medical treatment. In the visually impaired group, 23 $(14.5 \%)$ underwent surgery and $136(85.5 \%)$ received medical treatment. The main causes of blindness among the patients were cataract glaucoma, optic atrophy, hereditary retinal

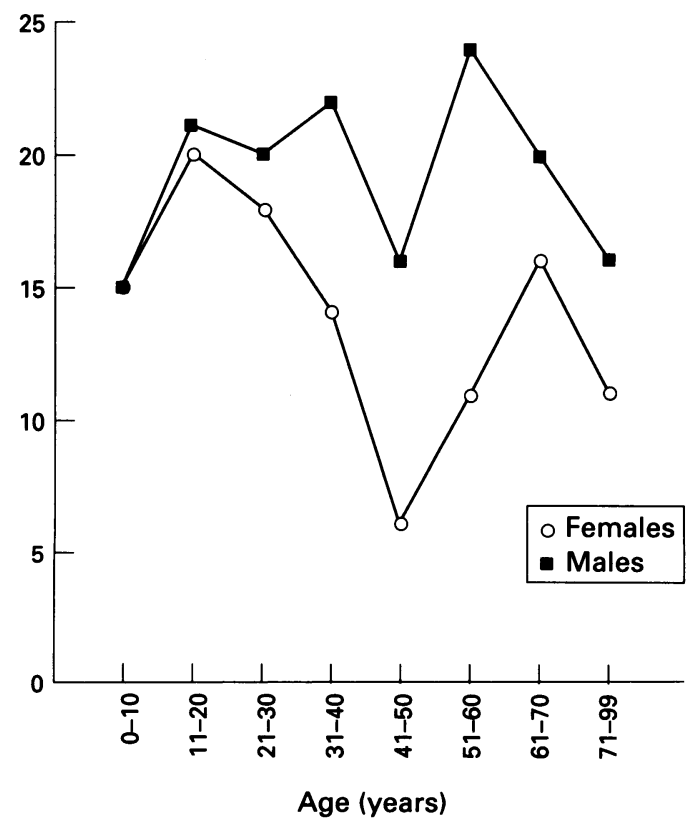

Figure 2 Distribution of blindness by age and sex $(n=265)$. diseases, trauma, optic neuritis, xerophthalmia, uveitis, retinal detachment, and diabetic retinopathy. Frequencies and percentages are presented in Table 1.

\section{ANATOMICAL SITE OF BLINDNESS}

\section{Anterior segment}

Cataracts and xerophthalmia totalled $28.3 \%$ of the blind group. When these diseases were considered together with uveitis $(3.0 \%)$, trauma affecting the anterior segment and ultimately conducive to blindness $(5.3 \%)$, and other cases $(6.0 \%)$, anterior segment pathology totalled $42 \cdot 6 \%$.

\section{Posterior segment}

Some $25 \cdot 3 \%$ of the cases of blindness were due to posterior segment damage - that is, retinal detachment, hereditary retinopathies, optic neuritis and atrophy, and diabetic retinopathy. Along with $1 \cdot 1 \%$ of cases of posterior uveitis and $6.4 \%$ of other pathologies, posterior pole damage was observed in $32.8 \%$ of the blind group.

\section{Whole eye}

In advanced glaucoma both anterior and posterior segments are affected. These cases, together with $2 \cdot 3 \%$ of the traumas affecting the whole eye and $1.5 \%$ of other pathologies, totalled $24.6 \%$ of the blind in which the anterior and posterior poles were compromised.

\section{PREVENTABLE AND TREATABLE BLINDNESS}

Cataracts and retinal detachment are treatable, and the two taken together accounted for $27 \cdot 4 \%$ of the blinding diseases. Xerophthalmia is a fully preventable disease. Glaucoma and trauma are partially preventable. Altogether, these accounted for $32.5 \%$ of the causes of blindness. 


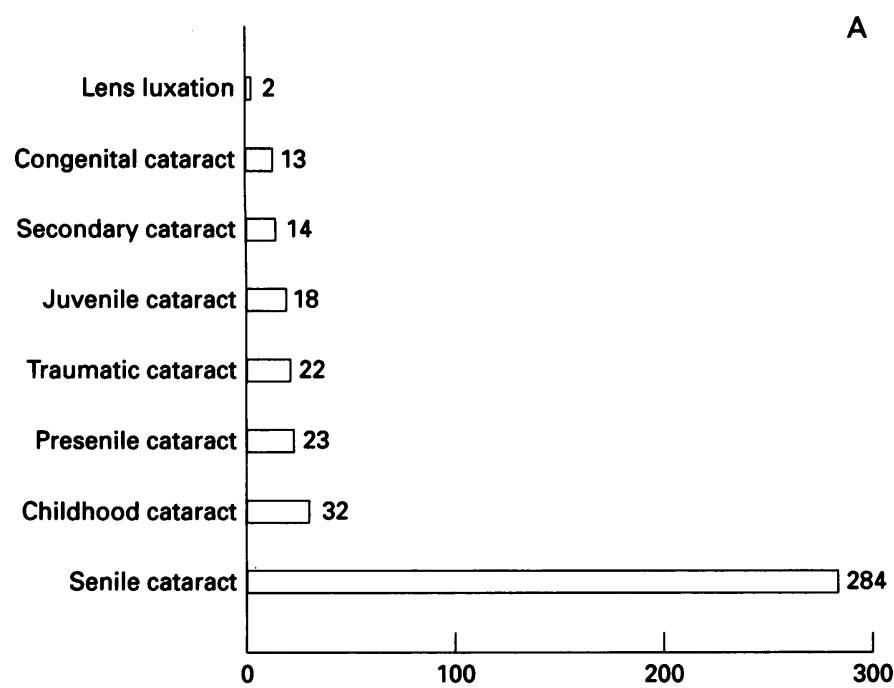

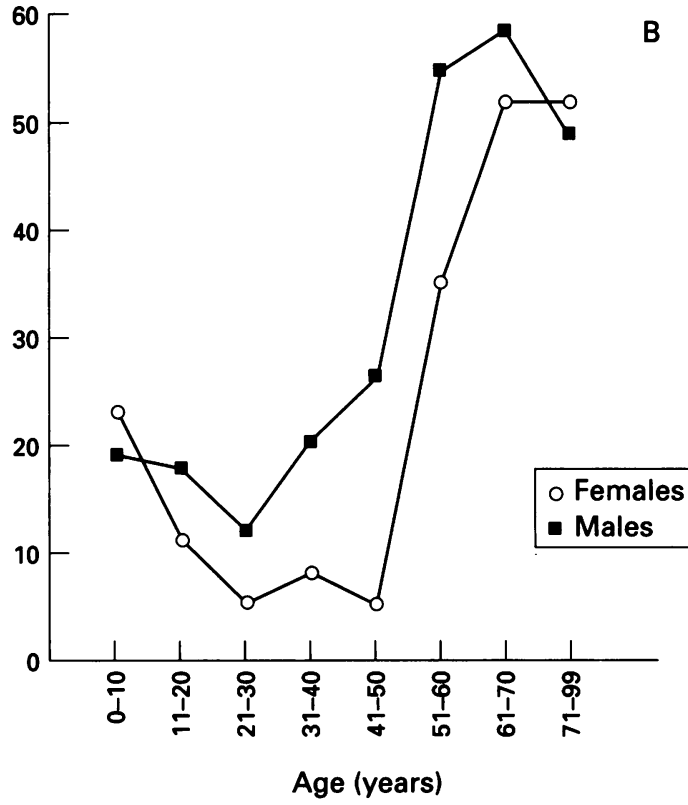

Age (years)

Figure 3 (A) Breakdown of lens diseases. Classification of primary cataracts depends on the age at which it was first noticed: congenital, less than 2 years; childhood, between 3 and 9 years; juvenile, between 10 and 30 years; presenile, between 31 and 45; senile, older than 45. Among traumatic cataracts, only sequelae of old traumas are included. Recent traumas affecting the lens are not included here. (B) Distribution of lens diseases by age and sex.

Some of the cases of uveitis could be preventable if dependent on endemic infectious diseases such as tuberculosis. Early treatment and close follow up of uveitis and diabetes mellitus could partly reduce $4.9 \%$ of the blind included in both groups.

\section{Discussion}

The Ophthalmic Institute was the only centre for the treatment of ophthalmic diseases open to civilians in Luanda at the time of the study. As only banal pathologies were discounted from the records (although they were always treated), the data presented here show some of the main diseases found in the population of the city, although no estimates of prevalence can be inferred. All hospital based data are subject to a number of biases; however, in the absence of a population based study, our data may, with some caution, be compared with the frequencies reported in surveys of ophthalmic pathologies in other African countries. The main purpose of the present study was to identify the main blinding diseases to be dealt with in ophthalmic healthcare projects in the
Angolan capital, which may also help in the planning of development aid programmes in Angola and other comparable countries (for example, Mozambique).

\section{CAUSES OF BLINDNESS}

The reported causes of blindness in Africa are, in order of importance: cataracts, trachoma, glaucoma, xerophthalmia, onchocerciasis, agerelated macular degeneration, diabetic retinopathy and leprosy. ${ }^{3}$ Reports from geographically similar countries such as Zimbabwe and Kenya also identify cataracts, corneal opacity, and open angle glaucoma as the main causes of blindness. ${ }^{4}$

Among our patients the main causes of blindness before treatment were: cataracts, glaucoma, optic nerve diseases (neuritis and atrophy), trauma, xerophthalmia, uveitis, hereditary retinal diseases (degenerative myopia, retinitis pigmentosa, albinism, and Stargardt's disease), retinal detachment, and diabetic retinopathy (Table 1, Fig 2).

In African adults, cataract is the principal cause of blindness. ${ }^{1}$ As in other reports,
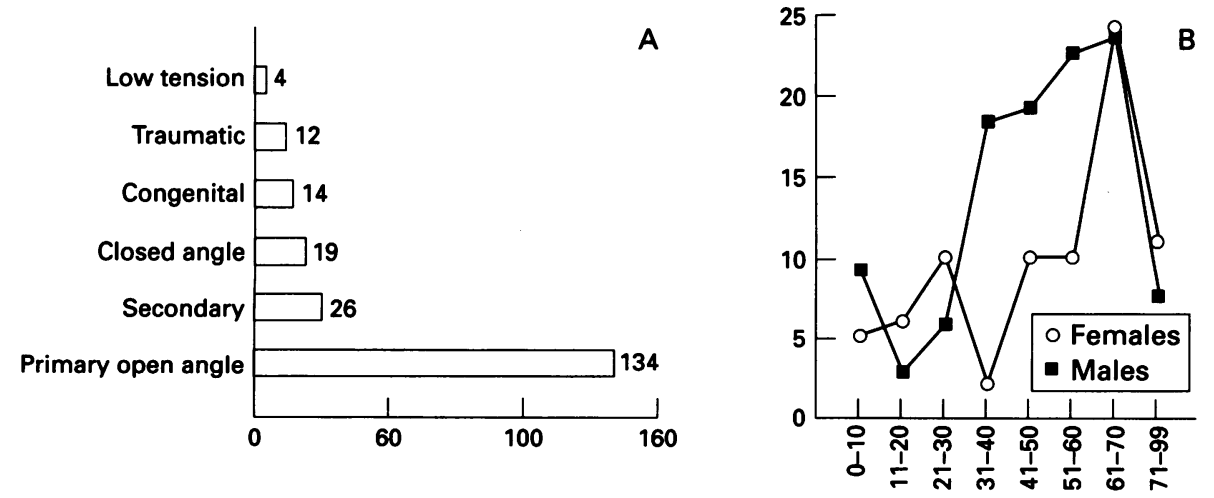

Age (years)

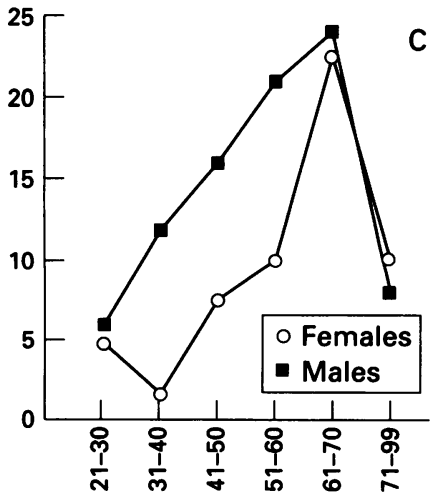

Age (years)

Figure 4 (A) Breakdown of glaucoma $(n=209)$. (B) Distribution of all glaucomas by age and sex $(n=209)$. (C) Distribution of primary open angle glaucoma by age and sex $(n=134)$. 


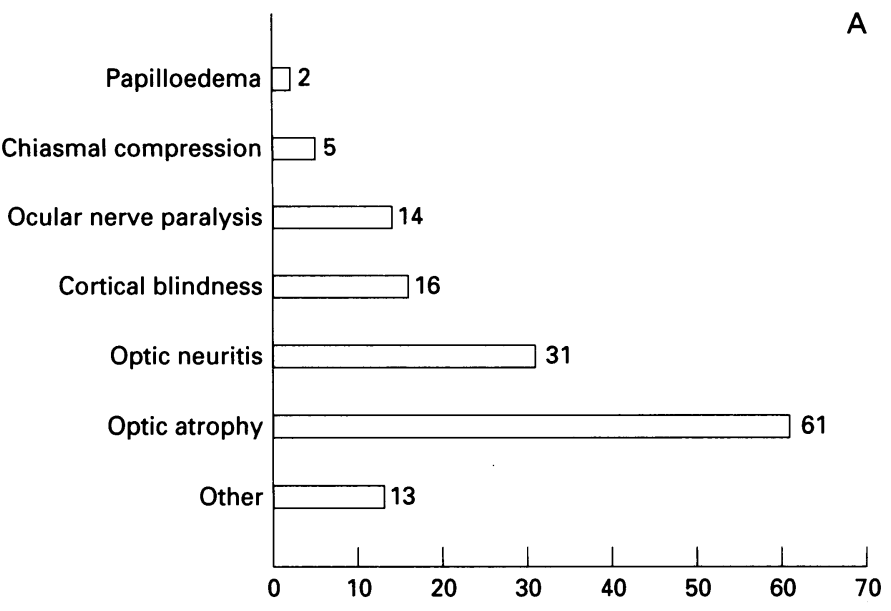

Figure 5 (A) Breakdown of neural diseases $(n=142)$. (B) Distribution of neural diseases by age and sex $(n=142)$.

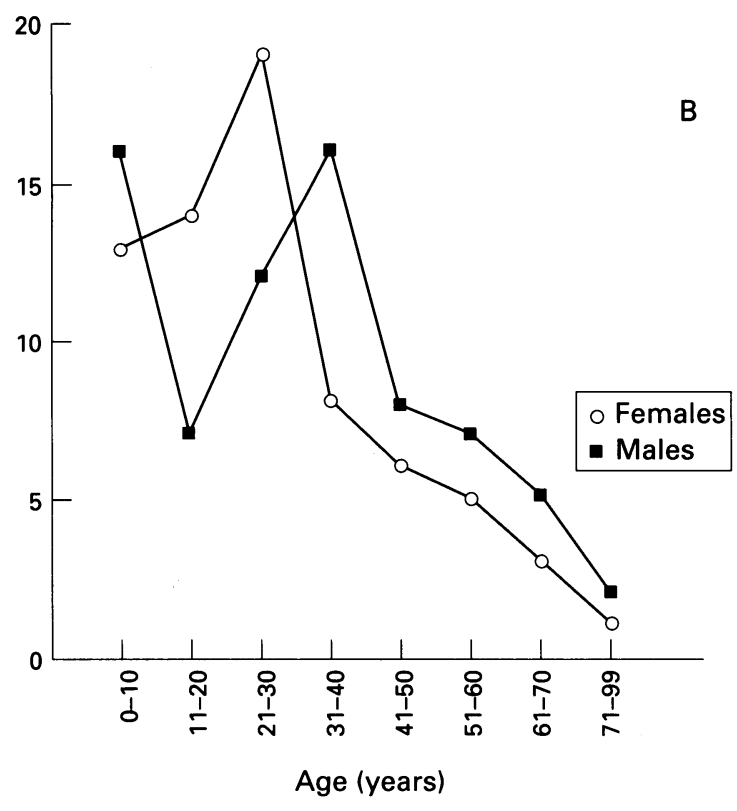

Age (years) cataracts were the main cause of bilateral blindness in our series. Training in cataract surgery was one of the main objectives of our programme. In children under 12 , cataract remains the chief cause of blindness (Fig 3).

Glaucoma is quoted as being a significant cause of blindness in hospital based reports in west Africa. ${ }^{5}$ In our records, glaucoma was the second most common cause of blindness. Congenital glaucoma in males was double that found in females. Figures $4 \mathrm{~A}$ and $\mathrm{B}$ show the distribution of all types of glaucoma by age and sex. Figure 4C shows the distribution of primary open angle glaucoma (POAG) by age and sex. Although middle aged females seem to be less affected by POAG, the number of glaucoma cases was equal in both sexes for the over 60s.

Optic atrophy and optic neuritis were the third major cause of bilateral blindness among our patients (Fig 5). Although it is listed in sixth place among the causes of bilateral blindness in black American patients, ${ }^{6}$ optic nerve disease is not reported as a major cause of blindness in other reports from African countries. ${ }^{4-9}$ Optic atrophy is listed in fourth place among aetiologies of childhood blindness in Africa. ${ }^{10}$ In Gambia, optic atrophy was found to be responsible for $2 \%$ of cases of blindness. ${ }^{11}$ Although these data and ours are not fully comparable, the high proportion of optic nerve disease in our series may point towards a high level of genetic, nutritional, or infectious susceptibility among the population of Luanda.

Trachoma blindness is frequently found in the arid regions of north Africa, the Sahel and the dry plains of east and central Africa. ${ }^{3}$ Trachoma is closely related to standard of living, and is currently a significant problem in underserved rural areas or urban slums. ${ }^{12}$ In Luanda we saw numerous cases of inclusion conjunctivitis but few cases of blinding
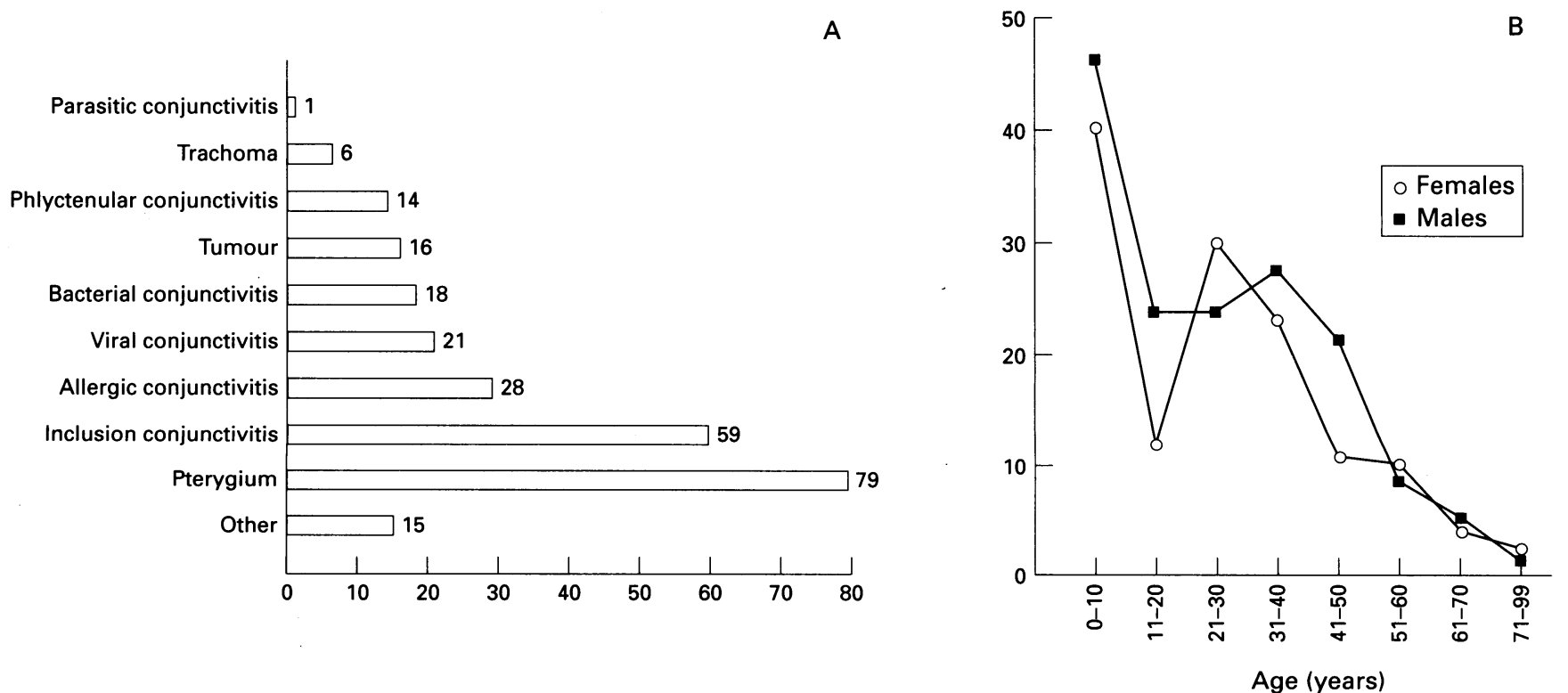

Figure 6 (A) Breakdown of conjunctival diseases $(n=258)$. Only a sample of the cases of inclusion conjunctivitis diagnosed were included in the records. Pterygium is included among conjunctival disorders. (B) Distribution of conjunctival diseases by age and sex ( $n=258)$. 

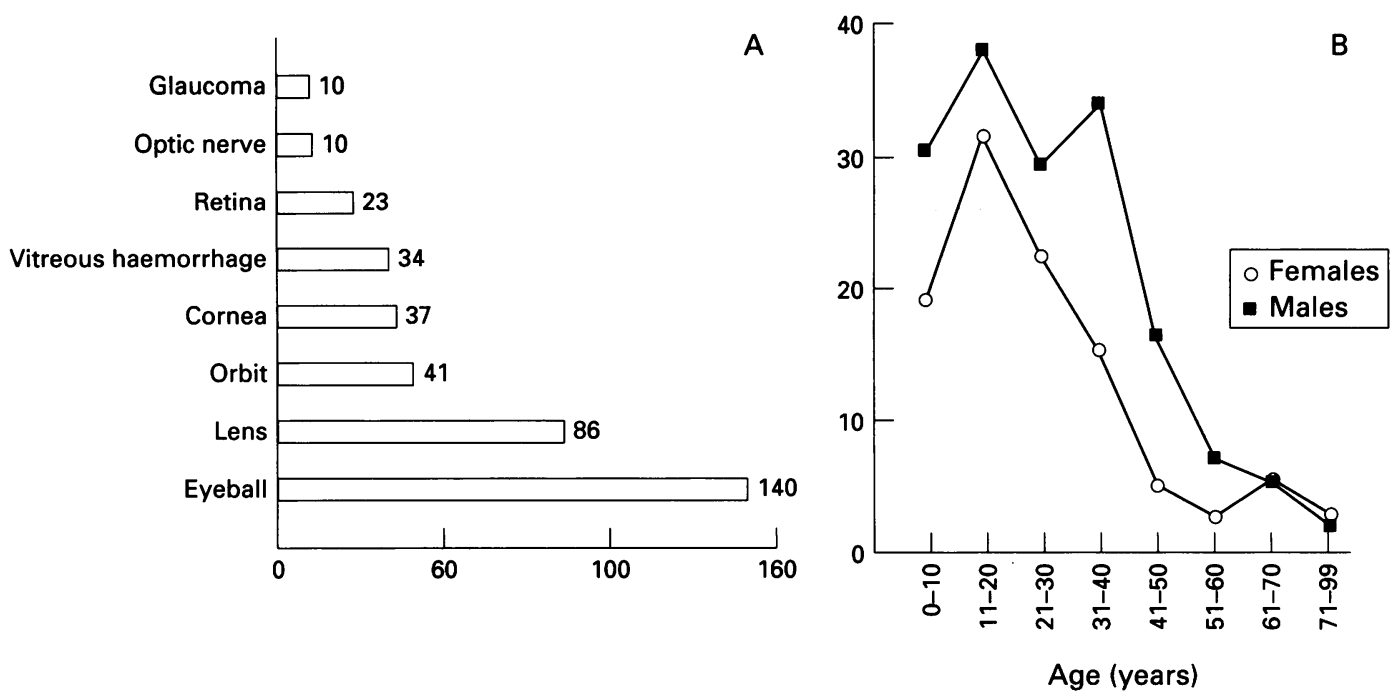

Figure 7 (A) Breakdown of traumatic diseases according to the anatomical structure involved $(n=380)$. (B) Distribution of injuries by age and sex.

trachoma. Inclusion conjunctivitis is endemic in Luanda, and present in children as well as adolescents and adults. Cases of this type of conjunctivitis treated in the emergency ward were so numerous that they were revised at the general hospital in order to avoid overcrowding at the institute. Only a few selected cases were referred to the institute, where they underwent a thorough examination. Although the number of cases reported here is only testimonial, all cases of true trachoma diagnosed in the emergency ward were treated at the institute. True trachoma seems to be rare in Luanda, accounting for only $2 \%$ of our cases of conjunctivitis. A breakdown of conjunctival cases is given in Figure 6. Pterygia is included among conjunctival disorders. Its frequency appears to be higher than that of inclusion conjunctivitis since the residents frequently referred cases of pterygia to the institute for surgery.

Trauma is the fourth most common cause of bilateral blindness in our data. The importance of trauma in unilateral blindness is even greater. Not only does the country suffer the effects of violent conflict, but personal attacks on civilians are everyday occurrences, since the perpetrators are never prosecuted. A breakdown of types of traumatism is given in Figure 7. 'Eyeball' indicates rupture of the eyeball by blunt trauma and is the most frequent form of traumatism. Adolescents are the most seriously affected group, and young females are almost as frequently involved as males. A potentially fatal blow to the eye is a common method of punishment used by abusive males on their female partners. Trauma was found to be the second most prevalent blinding condition in the Kenya Rural Blindness Prevention Project. ${ }^{13}$

Vitamin A deficiency and measles are responsible for more than half the childhood blindness in Africa. ${ }^{3}$ In our series, xerophthalmia was ranked in fifth place for all age groups. Among children under 6, xerophthalmia and cataracts share first place. In our records, xerophthalmia was found in undernourished children affected by measles or acute respiratory disease, usually accompanied by diarrhoea. Corneal disease, when present, was often severe in such cases.
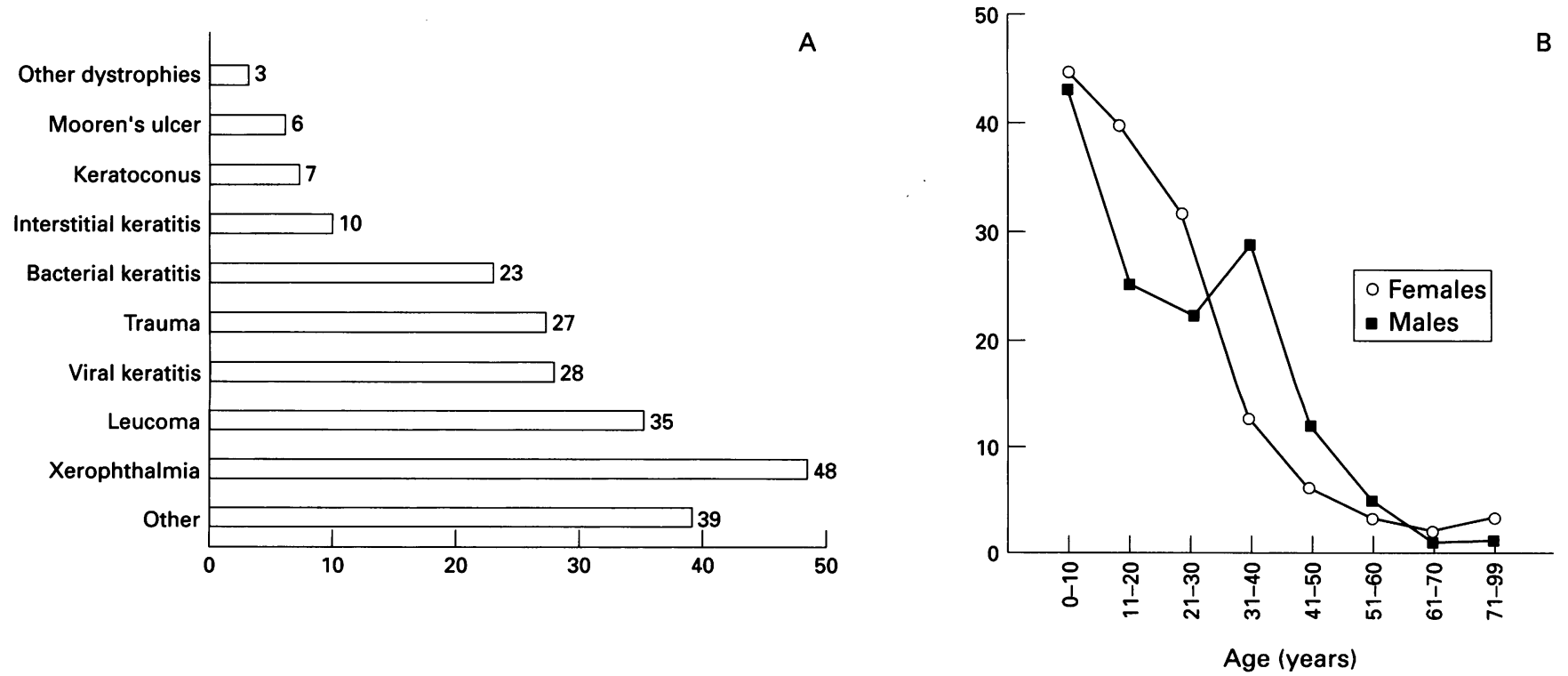

Figure 8 (A) Breakdown of corneal diseases $(n=226)$. (B) Distribution of corneal diseases by age and sex. 


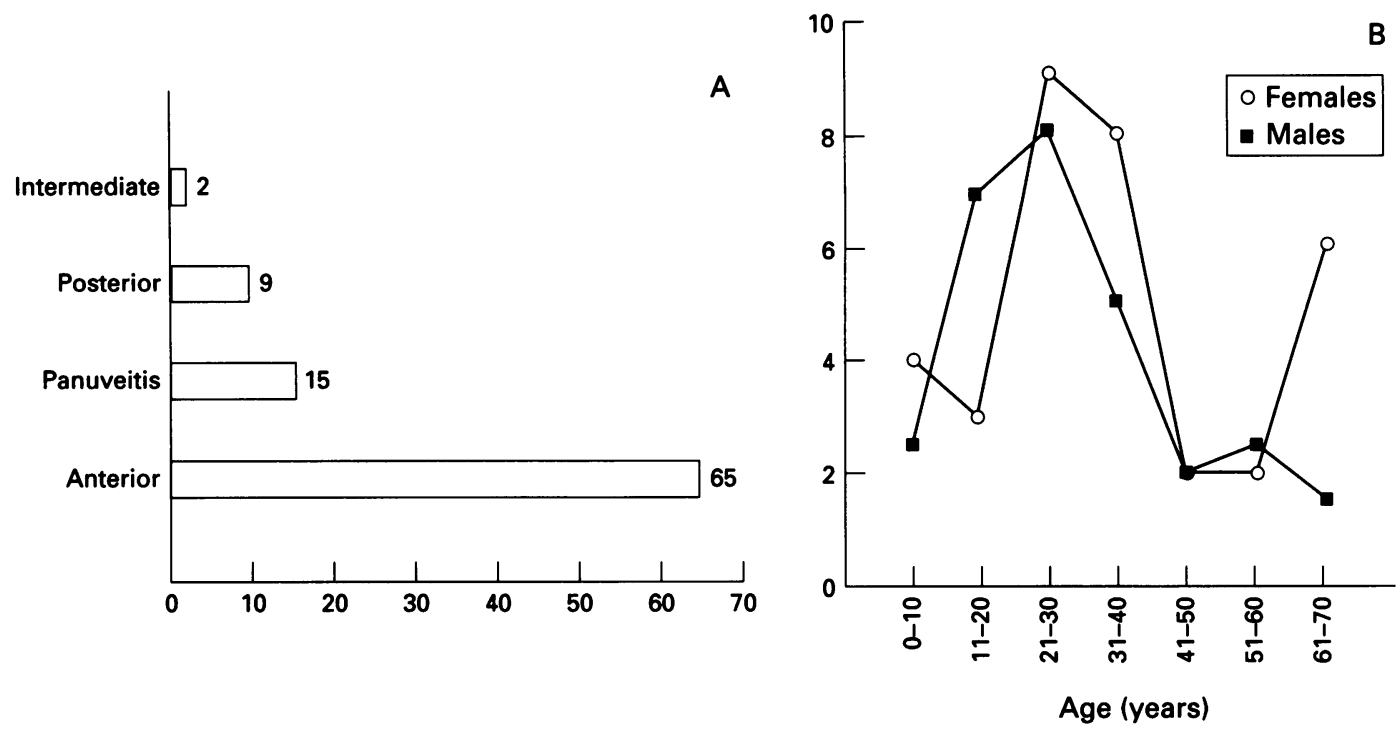

Figure 9 (A) Breakdown of uveal diseases based on the location of the principal lesion. (B) Distribution of uveal diseases by age and sex $(n=91)$

Corneal ulcers are associated with a rapid decrease in vitamin $\mathrm{A}$ reserves in the debilitated organism. ${ }^{14}$ Angola is a producer of palm oil, the richest natural source of vitamin A precursors, which would explain why chronic nutritional xerophthalmia is absent from our series. The main corneal pathology diagnosed at the institute is shown in Figure 8.

Uveitis is the sixth most common cause of blindness in our study. Only cases of active uveitis at the time of diagnosis were included in this category. Sequelae of probable uveitis origin, but not diagnosed by us where active, are not included here, but rather under the affected tissue (for example, secondary cataract). Maximal incidence of active uveitis was found among young adults $(75 \%$ were under 30 ). A large number of patients $(58 \%$ of granulomatous uveitis) gave a positive tuberculin skin test. The incidence of active tuberculosis in the province of Luanda is high: official 1990 figures estimated it at 41 new cases per 100000 inhabitants. ${ }^{15}$ Onchocerciasis is a major cause of blindness in west and central Africa. ${ }^{12}$ Although northern Angola falls within the limits of the endemic area, Luanda lies in the semiarid coastal region, and only one case with active filariasis was diagnosed in our centre. Distribution of uveitis according to its primary location is shown in Figure 9.

A group of congenital retinal degenerations share seventh place among the most common causes of blindness in our data (Fig 10). Degenerative myopia is by far the most important of the group. Age-related macular degeneration (ARMD) is in tenth place within this group, and is less frequent than retinitis pigmentosa and 'bull's eye maculopathy'. ARMD was not quoted as an important cause of blindness among black Americans in the Baltimore Eye Survey ${ }^{6}$; nor was it explicitly mentioned in studies of prevalence of ophthalmic diseases in Gambia and Kenya. ${ }^{2} 11$

The diagnosis of retinal diseases at the Ophthalmic Institute was made in the absence of electrophysiological tests. In a country where family diseases are almost never known

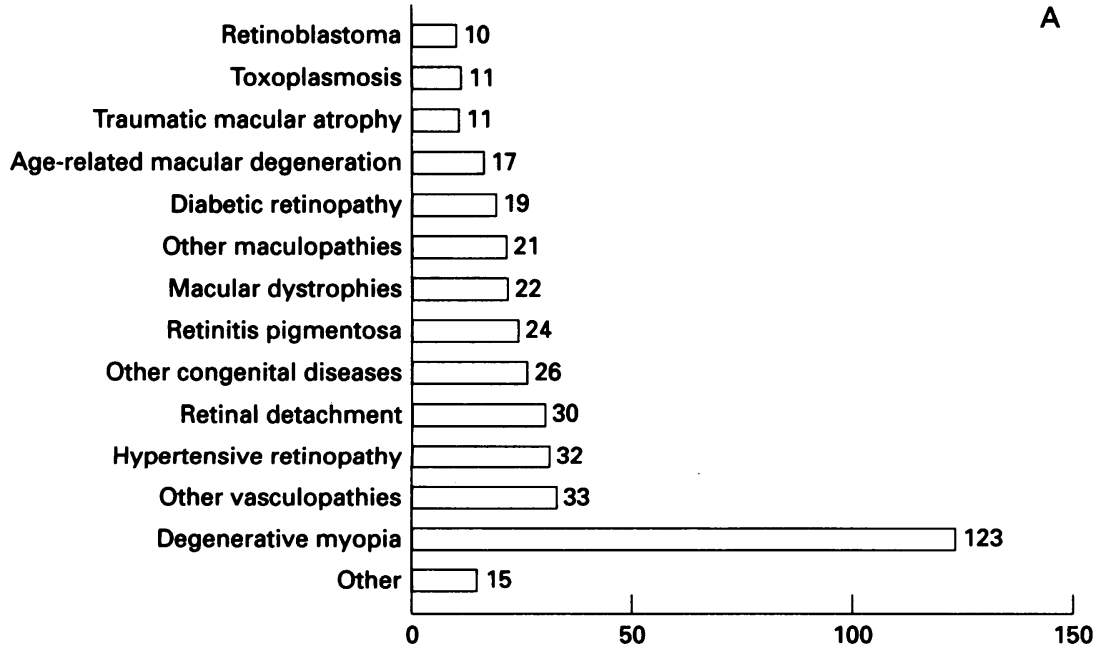

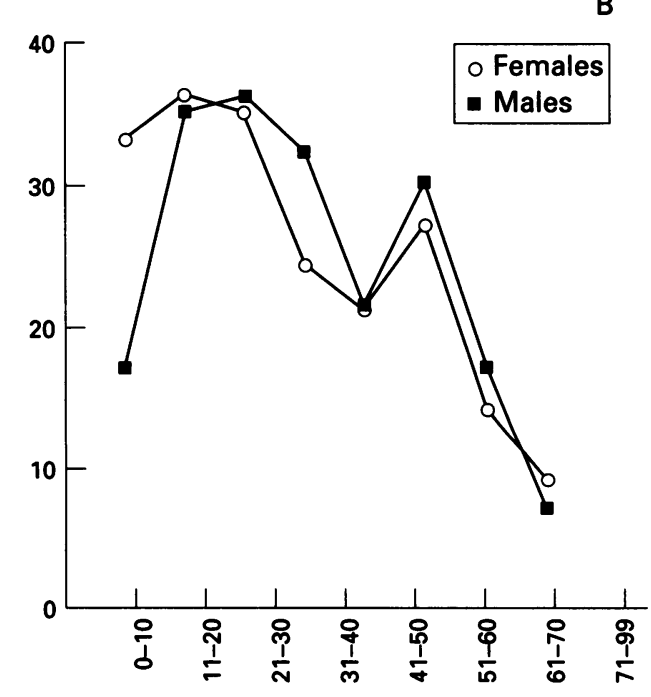


to the patients and where the massive consumption of self prescribed antimalaria drugs is widespread, the discrimination between family and acquired diseases is precarious. The diagnosis of bull's eye maculopathy remained merely descriptive.

\section{SOCIAL ASPECTS OF BLINDNESS}

Distribution of blindness by age shows a bimodal curve, with the modes at 15 and 60 years (Fig 2). An increase in mortality has been found to be associated with blindness in rural Africa (central Tanzania), especially among women. ${ }^{16}$ The lower number of cases of blindness among middle aged people treated at the institute may reflect the reduced chances of survival for the young blind in the Angolan environment. Among the young blind, the higher frequency of fatal cases of trauma in young males seems to be counterbalanced by the higher frequency of blinding optic nerve disorders in young females. The nature of optic neuritis and atrophy in this environment is uncertain. As the disease does not respond to treatment, and because of its importance as a blinding disease, especially among young people, any programme which attempted to ascertain the causes would be worth pursuing and preventive measures could be of considerable help.

Retinal detachment surgery requires early identification in order for the treatment to be effective, which largely depends on patients recognising the early signs and promptly seeking help. Here, as in other painless blinding diseases, such as silent uveitis, sanitary education of the population is of great importance. Glaucoma is preventable with early diagnosis but, being symptom free, demands a population wide programme for its early detection.

Sanitary education is also the key to the prevention of xerophthalmia, as the necessary resources are readily available in the country. The large number of cases of blindness caused by traumatism, normally the result of common assaults, highlights the importance of social education.

The importance of cataracts in all age groups cannot be overestimated. There is an urgent need to train Angolan ophthalmologists in extracapsular lens extraction techniques, although intraocular lens implants might not be considered as appropriate treatment in developing countries. ${ }^{1}$

Angola suffers a considerable deficit of sanitary services, including ophthalmological care. In order to optimise the available resources, a social education campaign ought always to complement any medical programme.

1 Foster A. Cataract blindness in Africa. Int Ophthalmol 1987; 18: $384-8$.

2 Loewenthal $\mathrm{R}, \mathrm{Pe}$ 'er J. A prevalence survey of ophthalmic diseases among the Turkana tribe in north-west Kenya. BrF Ophthalmol 1990; 74: 84-8.

3 Foster A, Johnson GJ. Magnitude and causes of blindness in the developing world. Int Ophthalmol 1990; 14: 135-40.

4 Schwab L. Ophthalmology in Zimbabwe. Arch Ophthalmol 1989; 107: 284

5 Wormald R, Foster A. Clinical and pathological features of chronic glaucoma in North-East Ghana. Eye 1990; 4: 107-14.

6 Sommer A, Tielsch JM, Royall RW, Witt KA, Ezrine S. Racial differences in the cause-specific prevalence of blindness in East Baltimore. N Engl $\mathcal{F}$ Med 1991; 325: 1412-7.

7 Adamson I, Taylor HR. Major causes of world blindness their treatment and prevention. Curr Opin Ophthalmo 1990; 1: 635-42.

8 Thompson JR, Rosenthal AR. Epidemiology of world blindness. Curr Opin Ophthalmol 1990; 1: 649-53.

9 Searle AET, Fielder AR. Screening for ocular disease and visual impairment. Curr Opin Ophthalmol 1990; 1: 654-9.

10 Kagame K, Schwab L. Childhood blindness: Dateline Africa. Ophthalmic Surg 1989; 20: 128-31.

11 Faal H, Minassian D, Sowa S, Foster A. National survey of blindness and low vision in the Gambia: results. $\mathrm{Br} \mathcal{f}$ Ophthalmol 1989; 73: 82-7.

12 Thylefors B, Négrel AD, Pararajasegaram R. Epidemiologic aspects of global blindness prevention. Curr Opin Ophthalmol 1992; 3: 824-34.

13 Whitfield R, Schwab L, Ross-Degnan D, Steinkuller P, Swartwood J. Blindness and eye disease in Kenya: ocular status survey results from the Kenya Rural Blindness Prevention Project. Br $\mathcal{F}$ Ophthalmol 1990; 74: 333-40.

14 Sommer A, Muhilal H. Nutritional factors in corneal xerophthalmia and keratomalacia. Arch Ophthalmol 1982; 100: 399-403.

15 Relatório anual de vigilancia epidemiologica de 1990. Delegaçao Provincial de Saúde de Luanda. Direcçao Provincial de Saúde Pública e Control de Endemias. República Popular de Angola: Ministerio da Saúde, 1991.

16 Taylor HR, Katala S, Munoz B, Turner V. Increase in mortality associated with blindness in rural Africa. Bull WHO 1991; 69: 335-8. 